\title{
Emerging 2D Nanomaterials for Multimodel Theranostics of Cancer
}

\author{
Wei Zhu ${ }^{1 * t}$, Helin $\mathrm{Li}^{2,3 t}$ and Peng Luo ${ }^{4 *}$ \\ ${ }^{1}$ Key Laboratory of Advanced Textile Materials and Manufacturing Technology and Engineering Research Center for Eco-Dyeing \\ and Finishing of Textiles, Ministry of Education, Zhejiang Sci-Tech University, Hangzhou, China, ${ }^{2}$ Collaborative Innovation Center \\ of Yangtze River Delta Region Green Pharmaceuticals, Zhejiang University of Technology, Hangzhou, China, ${ }^{3}$ Institute of \\ Technical and Macromolecular Chemistry, RWTHAachen University, Aachen, Germany, ${ }^{4}$ Department of Orthopedic Trauma, The \\ Second Affiliated Hospital and Yuying Children's Hospital of Wenzhou Medical University, Wenzhou, China
}

Keywords: 2D nanomaterials, nanomedicine, theranostics, clinical, cancer

\section{INTRODUCTION}

Cancer is a major public health problem worldwide nowadays, with more than 18 million new cases each year. In 2020, the diagnosis and treatment of cancer were interfered by the coronavirus disease 2019 (COVID-19) pandemic. Reduced access to care resulted in delays in diagnosis and treatment in relation to increased death (Li et al., 2021b; Siegel et al., 2021).

OPEN ACCESS

Edited by:

Masoud Mozafari,

University of Toronto, Canada

Reviewed by:

Xianwen Wang,

Anhui Medical University, China

Zhen Yuan,

University of Macau, China

*Correspondence:

Wei Zhu

willian_fox@szu.edu.cn

Peng Luo

luopeng@wmn.edu.cn

${ }^{t}$ These authors have contributed equally to this work

Specialty section:

This article was submitted to Biomaterials,

a section of the journal Frontiers in Bioengineering and Biotechnology

Received: 01 September 2021 Accepted: 18 October 2021 Published: 19 November 2021

Citation:

Zhu W, Li H and Luo P (2021) Emerging 2D Nanomaterials for Multimodel Theranostics of Cancer. Front. Bioeng. Biotechnol. 9:769178. doi: 10.3389/fbioe.2021.769178
Although cancer treatment strategies were developed, it is still extremely important to speed up the diagnosis and treatment of cancer. Recently, theranostics have stimulated increased attention in both research and clinical fields, which allow very intelligent diagnostic imaging ability with therapeutic intervention within spatial colocalization (Li et al., 2017b; Kang et al., 2020; Li et al., 2020). Up to now, various theranostic systems have been explored, involving different modalities of diagnosis and therapies. To gain versatility, increasingly complex nanoparticles are designed to enable multimodal imaging and combination therapy (Li et al., 2017a; Chen et al., 2018). However, the purpose brings the difficulty of nanomaterials with a great deal of uncertainty, which seriously hampers clinical progress. For clinical transformation, the key is to achieve image-mediated therapy with the simpler components of nanomaterials (Li H. et al., 2019; Li et al., 2019b; Li et al., 2021c; Li et al., 2021d). Over the last few years, two-dimensional (2D) nanomaterials have been widely used for cancer diagnosis and treatment with the design based on simple components (Figure 1) (Wang and Cheng, 2019; Cheng et al., 2020; Wang et al., 2021).

Compared with other nanomaterials, e.g., liposome, dendrimer, and carbon nanotube, 2D nanomaterials have unique advantages that enable them to be requested as a biomedicine so conveniently (Gazzi et al., 2019; Gravagnuolo et al., 2021). Firstly, the rich source of 2D nanomaterials provides plentiful resources to meet different requirements for applications, including hexagonal boron nitride, group-VA semiconductors, graphitic carbon nitride, transition metal carbides, and transition metal dichalcogenides. Second, the good chemical, physical, and biological properties of $2 \mathrm{D}$ nanomaterials, such as optical, magnetic, electrical, or catalytic properties, can be well matched to provide desirable performance for diagnostics, imaging, or therapy of cancer that can be applied in the fields of practical biomedical applications. Third, the preparation of $2 \mathrm{D}$ nanomaterials is relatively simple with good yields in the laboratory (Zhang et al., 2021). The feasibility of 2D nanomaterials points out that they can be developed as promising clinical nanoplatforms for cancer theranostics (Huang et al., 2021).

Herein, the recent processes of the synthesis and applications of $2 \mathrm{D}$ nanomaterials for the treatment and diagnosis of cancer were discussed and summarized. Based on the large surface area and exceptional physicochemical properties, the various kinds of 2D nanomaterials were developed in the field of nanomedicine due to their excellent multimodal-imaging-guided synergetic cancer 


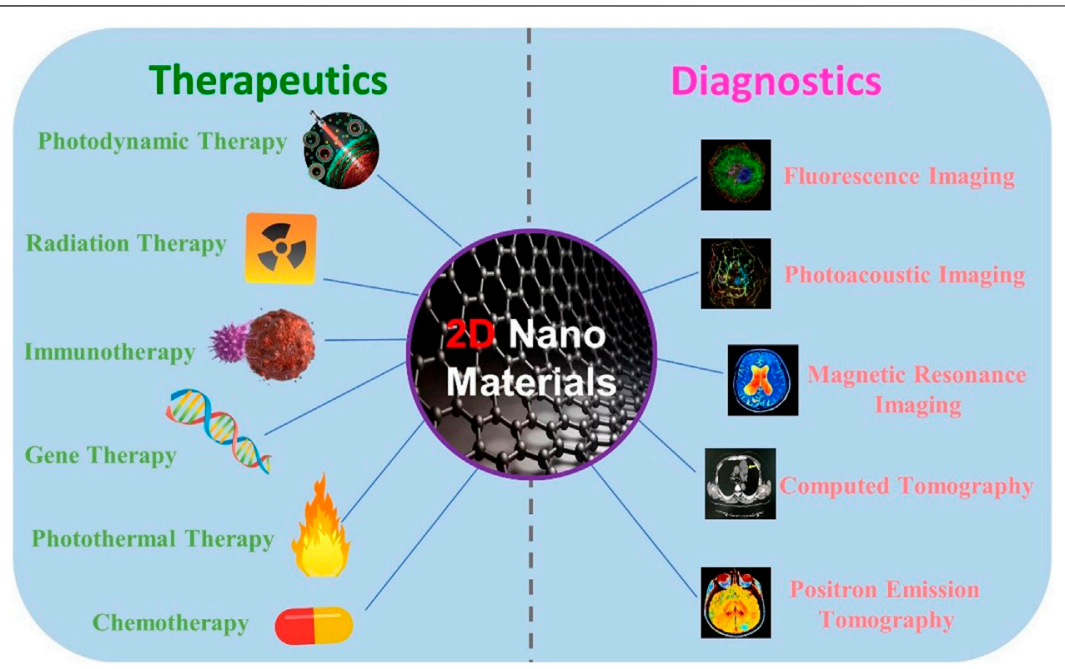

FIGURE 1 | Treatment and diagnosis methods based on the combined 2D nanomaterials. Some elements are adapted with permissions from Wang et al. (2018), copyright 2018 Ivyspring International Publisher.

TABLE 1 | Classification and applications of 2D material used for multimodel theranostics of cancer.

\begin{tabular}{|c|c|c|c|c|}
\hline 2D material type & Treatment means & Imaging method & Cancer type & References \\
\hline $\mathrm{GO}^{\mathrm{a}}$ & CHT, РTा & $\mathrm{FI}$ & Lymph cancer & Sun et al. (2008) \\
\hline GO & CHT, GT, PTT & FI, PET, CT & Breast cancer & Yang et al. (2013) \\
\hline GO & PDT, PTा & $\mathrm{FI}$ & Oral cancer & Tian et al. (2011) \\
\hline GO & $\mathrm{RT}, \mathrm{PTT}$ & CT, X-ray & Breast cancer & Chen et al. (2015) \\
\hline GO & $\mathrm{IT}, \mathrm{PTT}$ & $\mathrm{FI}$ & Colon cancer & Yan et al. (2019) \\
\hline TMDs $^{\mathrm{b}}\left(\operatorname{ReS}_{2}\right)$ & RT, PTT & $\mathrm{CT}, \mathrm{PAl}$ & Breast cancer & Qian et al. (2015) \\
\hline TMDs $\left(\mathrm{MoS}_{2}\right)$ & CHT, PTा & $\mathrm{FI}$ & Breast cancer & Liu et al. (2014) \\
\hline TMDs $\left(\mathrm{MoS}_{2}\right)$ & GT, PTा & $\mathrm{FI}$ & Rectal cancer & Kim et al. (2016) \\
\hline TMDs $\left(\mathrm{MoS}_{2}\right)$ & $\mathrm{IT}, \mathrm{PTT}$ & $\mathrm{FI}$ & Leukemia & Han et al. (2017) \\
\hline TMDs $\left(\mathrm{WS}_{2}\right)$ & $\mathrm{PTT}, \mathrm{RT}$ & PAI, CT, MRI & Breast cancer & Cheng et al. (2015) \\
\hline Mxenes $\left(\mathrm{Ti}_{3} \mathrm{C}_{2}\right)$ & PTा & $\mathrm{FI}$ & Breast cancer & Lin et al. (2017b) \\
\hline Mxenes $\left(\mathrm{Ta}_{4} \mathrm{C}_{3}\right)$ & РTा & PAI, CT & Breast cancer & Lin et al. (2018) \\
\hline Mxenes $\left(\mathrm{Nb}_{2} \mathrm{C}\right)$ & PTा & PAl & Breast cancer & Lin et al. (2017a) \\
\hline $\mathrm{BP}^{\mathrm{C}}$ & PDT & $\mathrm{FI}$ & Cervical cancer & Lv et al. (2016) \\
\hline $\mathrm{BP}$ & $\mathrm{CHT}$ & $\mathrm{FI}$ & Cervical cancer & Tao et al. (2017) \\
\hline $\mathrm{LDHs}^{\mathrm{d}}$ & CHT, PTT, PDT & $\mathrm{FI}$ & Liver cancer & Peng et al. (2018) \\
\hline LDHs & $\mathrm{CHT}, \mathrm{GT}$ & $\mathrm{FI}$ & Breast cancer & Li et al. (2014a) \\
\hline 2D $\mathrm{MOF}^{\mathrm{e}}$ & PDT, PTा & $\mathrm{MRI}$ & Osteosarcoma & Li et al. (2018) \\
\hline $\mathrm{hBN}^{f}$ & $\mathrm{CHT}$ & $\mathrm{FI}$ & Prostate cancer & Weng et al. (2014) \\
\hline
\end{tabular}

${ }^{a} \mathrm{GO}$, graphene oxide.

${ }^{b}$ TMDs, transition metal dichalcogenides.

${ }^{c} B P$, black phosphorous.

${ }^{d} \mathrm{LDH}$, layered double hydroxides.

${ }^{e} \mathrm{MOF}$, metal-organic frameworks.

${ }^{t} h B N$, hexagonal boron nitride.

therapy performance. Moreover, 2D nanomaterials also can be developed in the field of cancer immunotherapy through encapsulating bioinspired cell membranes for cancer-targeting therapy and, thus, provides an advance in personalized immunotherapy. Encouragingly, a safe and efficient 2D nanomaterial platform has been reported to realize the clinical nanomedicines with excellent efficacy of survival rate of $100 \%$ in vivo without preparing the complex nanoplatforms.

\section{DISCUSSION}

Owing to the large surface area and exceptional physicochemical properties of 2D nanomaterials, 2D nanomaterial-based multifunctional nanocomposites are promising materials for multimodal-imaging-guided synergetic cancer therapy (Table 1) (Zhong et al., 2021). A lot of theranostic platforms have been developed, involving diagnosis [computed tomography (CT), 
fluorescence imaging (FI), magnetic resonance imaging (MRI), photoacoustic imaging (PAI), positron emission tomography (PET), etc.] and therapies [chemotherapy $(\mathrm{CHT})$, photothermal therapy (PTT), photodynamic therapy (PDT), radiation therapy (RT), gene therapy (GT), immunotherapy (IT), etc.] (Li X. et al., 2014; Li et al., 2016; Xing et al., 2020). Multimodal is far superior to single-component imaging and diagnosis, which are complementary and enhance each other. Taking triple-modal PAI/MRI/CT as examples, the strong nearinfrared (NIR) absorbance of $\mathrm{WS}_{2}$ with high photothermal conversion efficiency enables PA imaging; the $\mathrm{WS}_{2}$ nanosheet-doped $\mathrm{Gd}^{3+}$ ions offer a strong contrast in T1-weighted MR imaging. Meanwhile, W and Gd elements could attenuate X-ray irradiation to allow for CT imaging (Li X. et al., 2014). Graphene is a 2D layer of carbon atoms that can be used in a wide range of applications including nanomedicine (Tufano et al., 2020). Owing to its extremely large specific surface areas, graphene has great potential in biosensors, drug delivery, and cancer treatment (Gu et al., 2019). Bianco and co-workers recently introduced a multifunctional drug delivery platform based on graphene for cancer therapy applications (Lucherelli et al, 2020). The multifunctional graphene platform, modified with indocyanine green as the fluorophore, folic acid as the targeting agent to Hela cells, and doxorubicin (DOX) as the therapeutic molecule, showed a combined synergistic effect of targeting drug release of DOX for selectively killing cancer and photothermal properties under NIR irradiation. A significant reduction of Hela cell viability was observed, suggesting that the nanoplatform has been proven for effective anticancer therapy attributed to the synergistic effect of chemo- and photothermal therapies. Moreover, due to its good biocompatibility and biodegradability, black phosphorus nanoparticles have attracted more and more attention in the biomedical field in recent years (Zhang et al., 2021). Tang and his colleagues demonstrated a facile method to construct a new aggregation-induced emission (AIE) photosensitizer combined with 2D black phosphorus nanosheets and their application for multimodal theranostics involving NIR fluorescence-photothermal dual imagingguided synergistic photodynamic-photothermal therapy (Huang et al., 2020). With high stability and good biocompatibility, the hybrid nanomaterial can effectively generate reactive oxygen species and show bright NIR fluorescence and excellent photothermal conversion efficiency. It also exhibits the effective lysosomal escape and mitochondria targeting effects due to the amine groups that protonated at the acidic tumor microenvironment. These remarkable characteristics make it have enhanced antitumor efficacy to $4 \mathrm{~T} 1$ skin tumor. In recent years, cancer immunotherapy has begun to attract widespread attention, becoming an effective method in the clinical treatment of cancer. Through encapsulation with cell membranes, 2D materials have become popular in cancer immunotherapy that can be used as a biomimetic nanocarrier to load anticancer drugs for cancer-targeting therapy. Chen and his colleagues reported that bioinspired red blood cell (RBC) membrane is used for wrapping $2 \mathrm{D} \mathrm{MoSe}_{2}$ nanosheets with high photothermal conversion efficiency to achieve enhanced biocompatibility and circulation time (He et al., 2019). 2D $\mathrm{MoSe}_{2}$ nanosheets encapsulated with cell membranes has tumor-targeting capability. The combination of $\mathrm{RBC}-\mathrm{MoSe}_{2}$ with anti-PD-1 immunotherapy prevented the activation of the PD-1/PD-L1 pathway to avoid immune failure and stopped the transmission of an antiapoptotic signal to tumor cells, indicating the specific immune responses to CT 26 colorectal tumor. This $\mathrm{RBC}-\mathrm{MoSe}_{2}$-potentiated PTT demonstrated the efficient photothermal-potentiated systemic cancer immunotherapy via utilizing biomimetic $2 \mathrm{D}$ nanomaterial that was effectively able to kill cancer cells and, thus, provides potential advance for clinical translation.

Despite many studies of 2D nanomaterials used in theranostics of cancer, cases of conversion to the clinic are rarely reported. The recent article by Xing and fellow workers is both timely and exciting for 2D nanomaterial clinical translation (Li et al., 2021a). In this study, the a-tocopherol succinate (a-TOS)modified two-dimensional molybdenum disulfide $\left(\mathrm{MoS}_{2}\right)$ platform was successfully developed for collaborative computed tomography (CT)/photoacoustic (PA)/ photothermal imaging and selective chemotherapy of ovarian cancer. First, the platform has a safe irradiation dose, and its photothermal efficiency (65.3\%) is much higher than that of other photothermal materials (ICG = $3.1 \%$, cyanine dyes $=26.6 \%$, and gold nanorods $=21.0 \%$ ) (Jung et al., 2017). Moreover, the $\alpha$-TOS is introduced to the platform through a covalent link to realize the selective chemotherapy of cancer cells. The targeted ligand FA is used for specific targeting to achieve effective accumulation in tumor. Owing to good properties, the platform can completely cure solid tumors through photothermal therapy and then kill the remaining cancer cells by selective chemotherapy. The photothermal-selective chemotherapy platform exhibits a synergistic effect in tumor treatment. Moreover, the platform, as a control agent of cooperative CT/PA/ thermal images, is useful to achieve precise localization of tumor before performing combined therapy. Crucially, there were almost no side effects during the whole treatment. Its good efficacy and safety in vivo make mice survival rate reach $100 \%$ in 91 days. Remarkably, the platform can be biodegraded and metabolized in vivo. According to these latest clinical transformation concepts, a-TOS combines 2D MoS 2 as a promising treatment platform, which can be used to achieve convincing efficacy and safety benefits of cancer treatment.

\section{CONCLUSION}

In conclusion, due to their unique physical and chemical properties, $2 \mathrm{D}$ nanomaterials can be used as a platform to realize highly integrated imaging and treatment functions for various types of cancer. We presented the recent progress of the fabrication and studies of $2 \mathrm{D}$ nanomaterials, with particular attention on the viewpoints of multimodalimaging-guided synergetic cancer therapy and cancer immunotherapy. However, despite the reported exciting results, future clinical application of $2 \mathrm{D}$ nanomaterials still faces great challenges such as toxicity, low yield, and difficulties in clinical transition. In terms of clinical transformation, the main obstacle is the potential long-term safety of these nanomaterials, especially those nonbiodegradable nanomaterials that remain in the body for a long time. For future clinical application of 2D 
nanomaterials in the medical field, the following six aspects should be focused on: 1) potential untargeted toxicity from the material, which requires more systematic clinical testing; 2) selection of specific functional materials prior to specific types of cancer treatment; 3) functional optimization of materials; 4) the ongoing concern for the degradability of materials; 5) the imbalance between increasing nanomedicines and low clinical translation; and 6) a more biocompatible and biosafe nanoplatform. Although there are still many knowledge gaps in the field, virtuous perspectives for $2 \mathrm{D}$ nanomaterials were evidenced by remarkable progress in recent years. Therefore, 2D nanomaterials, especially those biodegradable nanomaterials, may indeed be a promising application of nanomedical systems in cancer treatment.

\section{REFERENCES}

Chen, H., Gu, Z., An, H., Chen, C., Chen, J., Cui, R., et al. (2018). Precise Nanomedicine for Intelligent Therapy of Cancer. Sci. China Chem. 61, 1503-1552. doi:10.1007/s11426-018-9397-5

Chen, L., Zhong, X., Yi, X., Huang, M., Ning, P., Liu, T., et al. (2015). Radionuclide ${ }^{131}$ I Labeled Reduced Graphene Oxide for Nuclear Imaging Guided Combined Radio- and Photothermal Therapy of Cancer. Biomaterials 66, 21-28. doi:10.1016/j.biomaterials.2015.06.043

Cheng, L., Wang, X., Gong, F., Liu, T., and Liu, Z. (2020). 2D Nanomaterials for Cancer Theranostic Applications. Adv. Mater. 32, 1902333. doi:10.1002/ adma.201902333

Cheng, L., Yuan, C., Shen, S., Yi, X., Gong, H., Yang, K., et al. (2015). Bottom-Up Synthesis of Metal-Ion-Doped $\mathrm{WS}_{2}$ Nanoflakes for Cancer Theranostics. ACS Nano 9, 11090-11101. doi:10.1021/acsnano.5b04606

Gazzi, A., Fusco, L., Khan, A., Bedognetti, D., Zavan, B., Vitale, F., et al. (2019). Photodynamic Therapy Based on Graphene and MXene in Cancer Theranostics. Front. Bioeng. Biotechnol. 7, 295. doi:10.3389/fbioe.2019.00295

Gravagnuolo, A. M., Morales-Narváez, E., and Martucci, A. (2021). Editorial: Biointerfacing 2D Nanomaterials and Engineered Heterostructures. Front. Bioeng. Biotechnol. 8, 639723. doi:10.3389/fbioe.2020.639723

Gu, Z., Zhu, S., Yan, L., Zhao, F., and Zhao, Y. (2019). Graphene-based Smart Platforms for Combined Cancer Therapy. Adv. Mater. 31, 1800662. doi:10.1002/adma.201800662

Han, Q., Wang, X., Jia, X., Cai, S., Liang, W., Qin, Y., et al. (2017). CpG Loaded $\mathrm{MoS}_{2}$ Nanosheets as Multifunctional Agents for Photothermal Enhanced Cancer Immunotherapy. Nanoscale 9, 5927-5934. doi:10.1039/C7NR01460K

He, L., Nie, T., Xia, X., Liu, T., Huang, Y., Wang, X., et al. (2019). Designing Bioinspired 2D MoSe ${ }_{2}$ Nanosheet for Efficient Photothermal-Triggered Cancer Immunotherapy with Reprogramming Tumor-Associated Macrophages. Adv. Funct. Mater. 29, 1901240. doi:10.1002/adfm.201901240

Huang, H., Feng, W., and Chen, Y. (2021). Two-dimensional Biomaterials: Material Science, Biological Effect and Biomedical Engineering Applications. Chem. Soc. Rev. 50, 11381-11485. Article in press. doi:10.1039/D0CS01138J

Huang, J., He, B., Zhang, Z., Li, Y., Kang, M., Wang, Y., et al. (2020). AggregationInduced Emission Luminogens Married to 2D Black Phosphorus Nanosheets for Highly Efficient Multimodal Theranostics. Adv. Mater. 32, 2003382. doi:10.1002/adma.202003382

Jung, H. S., Lee, J.-H., Kim, K., Koo, S., Verwilst, P., Sessler, J. L., et al. (2017). A Mitochondria-Targeted Cryptocyanine-Based Photothermogenic Photosensitizer. J. Am. Chem. Soc. 139, 9972-9978. doi:10.1021/jacs.7b04263

Kang, M., Zhang, Z., Song, N., Li, M., Sun, P., Chen, X., et al. (2020). Aggregationenhanced Theranostics: AIE Sparkles in Biomedical Field. Aggregate 1, 80-106. doi:10.1002/agt 2.7

Kim, J., Kim, H., and Kim, W. J. (2016). Single-Layered $\mathrm{MoS}_{2}$-PEI-PEG Nanocomposite-Mediated Gene Delivery Controlled by Photo and Redox Stimuli. Small 12, 1184-1192. doi:10.1002/smll.201501655

Li, B., Wang, X., Chen, L., Zhou, Y., Dang, W., Chang, J., et al. (2018). Ultrathin CuTCPP MOF Nanosheets: a New Theragnostic Nanoplatform with Magnetic

\section{AUTHOR CONTRIBUTIONS}

WZ and HL contributed equally to this work. WZ and PL discussed the concept of the manuscript. WZ and HL drafted the manuscript and performed the critical review of the manuscript. All authors reviewed the manuscript and approved the final version of the manuscript.

\section{FUNDING}

This article was partially supported by the China Scholarship Council and Wenzhou Municipal Science and Technology Bureau (Y2020057).

Resonance/near-Infrared thermal Imaging for Synergistic Phototherapy of Cancers. Theranostics 8, 4086-4096. doi:10.7150/thno.25433

Li, H., Li, X., Jain, P., Peng, H., Rahimi, K., Singh, S., et al. (2020). Dual-Degradable Biohybrid Microgels by Direct Cross-Linking of Chitosan and Dextran Using Azide-Alkyne Cycloaddition. Biomacromolecules 21, 4933-4944. doi:10.1021/ acs.biomac.0c01158

Li, H., Mergel, O., Jain, P., Li, X., Peng, H., Rahimi, K., et al. (2019a). Electroactive and Degradable Supramolecular Microgels. Soft Matter 15, 8589-8602. doi:10.1039/C9SM01390C

Li, L., Gu, W., Chen, J., Chen, W., and Xu, Z. P. (2014a). Co-delivery of siRNAs and Anti-cancer Drugs Using Layered Double Hydroxide Nanoparticles. Biomaterials 35, 3331-3339. doi:10.1016/j.biomaterials.2013.12.095

Li, X., Kong, L., Hu, W., Zhang, C., Pich, A., Shi, X., et al. (2021a). Safe and Efficient 2D Molybdenum Disulfide Platform for Cooperative Imaging-Guided Photothermal-Selective Chemotherapy: A Preclinical Study. J. Adv. Res. Article in press. doi:10.1016/j.jare.2021.08.004

Li, X., Li, H., Zhang, C., Pich, A., Xing, L., and Shi, X. (2021b). Intelligent Nanogels with Self-Adaptive Responsiveness for Improved Tumor Drug Delivery and Augmented Chemotherapy. Bioactive Mater. 6, 3473-3484. doi:10.1016/ j.bioactmat.2021.03.021

Li, X., Lu, S., Xiong, Z., Hu, Y., Ma, D., Lou, W., et al. (2019b). Light-Addressable Nanoclusters of Ultrasmall Iron Oxide Nanoparticles for Enhanced and Dynamic Magnetic Resonance Imaging of Arthritis. Adv. Sci. 6, 1901800. doi:10.1002/advs.201901800

Li, X., Ouyang, Z., Li, H., Hu, C., Saha, P., Xing, L., et al. (2021c). Dendrimerdecorated Nanogels: Efficient Nanocarriers for Biodistribution In Vivo and Chemotherapy of Ovarian Carcinoma. Bioactive Mater. 6, 3244-3253. doi:10.1016/j.bioactmat.2021.02.031

Li, X., Sun, H., Li, H., Hu, C., Luo, Y., Shi, X., et al. (2021d). Multi-Responsive Biodegradable Cationic Nanogels for Highly Efficient Treatment of Tumors. Adv. Funct. Mater. 31, 2100227. doi:10.1002/adfm.202100227

Li, X., Xing, L., Hu, Y., Xiong, Z., Wang, R., Xu, X., et al. (2017a). An RGDModified Hollow silica@Au Core/shell Nanoplatform for Tumor Combination Therapy. Acta Biomater. 62, 273-283. doi:10.1016/ j.actbio.2017.08.024

Li, X., Xing, L., Zheng, K., Wei, P., Du, L., Shen, M., et al. (2017b). Formation of Gold Nanostar-Coated Hollow Mesoporous Silica for Tumor Multimodality Imaging and Photothermal Therapy. ACS Appl. Mater. Inter. 9, 5817-5827. doi:10.1021/acsami.6b15185

Li, X., Xiong, Z., Xu, X., Luo, Y., Peng, C., Shen, M., et al. (2016). ${ }^{99 m}$ Tc-Labeled Multifunctional Low-Generation Dendrimer-Entrapped Gold Nanoparticles for Targeted SPECT/CT Dual-Mode Imaging of Tumors. ACS Appl. Mater. Inter. 8, 19883-19891. doi:10.1021/acsami.6b04827

Li, X., Yu, S., Yang, M., Xu, C., Wang, Y., and Chen, L. (2014b). Electronic Structure Analysis of glycine Oligopeptides and glycine-tryptophan Oligopeptides. Physica E: Low-dimensional Syst. Nanostructures 57, 63-68. doi:10.1016/ j.physe.2013.10.028

Lin, H., Gao, S., Dai, C., Chen, Y., and Shi, J. (2017a). A Two-Dimensional Biodegradable Niobium Carbide (MXene) for Photothermal Tumor 
Eradication in NIR-I and NIR-II Biowindows. J. Am. Chem. Soc. 139, 16235-16247. doi:10.1021/jacs.7b07818

Lin, H., Wang, X., Yu, L., Chen, Y., and Shi, J. (2017b). Two-dimensional Ultrathin MXene Ceramic Nanosheets for Photothermal Conversion. Nano Lett. 17, 384-391. doi:10.1021/acs.nanolett.6b04339

Lin, H., Wang, Y., Gao, S., Chen, Y., and Shi, J. (2018). Theranostic 2D Tantalum Carbide (MXene). Adv. Mater. 30, 1703284. doi:10.1002/adma.201703284

Liu, T., Wang, C., Gu, X., Gong, H., Cheng, L., Shi, X., et al. (2014). Drug Delivery with PEGylated MoS2Nano-Sheets for Combined Photothermal and Chemotherapy of Cancer. Adv. Mater. 26, 3433-3440. doi:10.1002/ adma.201305256

Lucherelli, M. A., Yu, Y., Reina, G., Abellán, G., Miyako, E., and Bianco, A. (2020). Rational Chemical Multifunctionalization of Graphene Interface Enhances Targeted Cancer Therapy. Angew. Chem. Int. Ed. 59, 14034-14039. doi:10.1002/anie.201916112

Lv, R., Yang, D., Yang, P., Xu, J., He, F., Gai, S., et al. (2016). Integration of Upconversion Nanoparticles and Ultrathin Black Phosphorus for Efficient Photodynamic Theranostics under $808 \mathrm{Nm}$ Near-Infrared Light Irradiation. Chem. Mater. 28, 4724-4734. doi:10.1021/ acs.chemmater.6b01720

Peng, L., Mei, X., He, J., Xu, J., Zhang, W., Liang, R., et al. (2018). Monolayer Nanosheets with an Extremely High Drug Loading toward Controlled Delivery and Cancer Theranostics. Adv. Mater. 30, 1707389. doi:10.1002/adma.201707389

Qian, X., Shen, S., Liu, T., Cheng, L., and Liu, Z. (2015). Two-dimensional TiS 2 Nanosheets for In Vivo Photoacoustic Imaging and Photothermal Cancer Therapy. Nanoscale 7, 6380-6387. doi:10.1039/C5NR00893J

Siegel, R. L., Miller, K. D., Fuchs, H. E., and Jemal, A. (2021). Cancer Statistics. CA Cancer J. Clin. 71, 7-33. doi:10.3322/caac.21654

Sun, X., Liu, Z., Welsher, K., Robinson, J. T., Goodwin, A., Zaric, S., et al. (2008). Nano-graphene Oxide for Cellular Imaging and Drug Delivery. Nano Res. 1, 203-212. doi:10.1007/s12274-008-8021-8

Tao, W., Zhu, X., Yu, X., Zeng, X., Xiao, Q., Zhang, X., et al. (2017). Black Phosphorus Nanosheets as a Robust Delivery Platform for Cancer Theranostics. Adv. Mater. 29, 1603276. doi:10.1002/adma.201603276

Tian, B., Wang, C., Zhang, S., Feng, L., and Liu, Z. (2011). Photothermally Enhanced Photodynamic Therapy Delivered by Nano-Graphene Oxide. ACS Nano 5, 7000-7009. doi:10.1021/nn201560b

Tufano, I., Vecchione, R., and Netti, P. A. (2020). Methods to Scale Down Graphene Oxide Size and Size Implication in Anti-cancer Applications. Front. Bioeng. Biotechnol. 8, 613280. doi:10.3389/fbioe.2020.613280

Wang, D., Lee, M. M. S., Xu, W., Kwok, R. T. K., Lam, J. W. Y., and Tang, B. Z. (2018). Theranostics Based on AIEgens. Theranostics 8, 4925-4956. doi:10.7150/thno. 27787
Wang, X., and Cheng, L. (2019). Multifunctional Two-Dimensional Nanocomposites for Photothermal-Based Combined Cancer Therapy. Nanoscale 11, 15685-15708. doi:10.1039/C9NR04044G

Wang, X., Zhong, X., Li, J., Liu, Z., and Cheng, L. (2021). Inorganic Nanomaterials with Rapid Clearance for Biomedical Applications. Chem. Soc. Rev. 50, 8669-8742. doi:10.1039/D0CS00461H

Weng, Q., Wang, B., Wang, X., Hanagata, N., Li, X., Liu, D., et al. (2014). Highly Water-Soluble, Porous, and Biocompatible boron Nitrides for Anticancer Drug Delivery. ACS Nano 8, 6123-6130. doi:10.1021/nn5014808

Xing, L., Li, X., Xing, Z., Li, F., Shen, M., Wang, H., et al. (2020). Silica/gold Nanoplatform Combined with a Thermosensitive Gel for Imaging-Guided Interventional Therapy in PDX of Pancreatic Cancer. Chem. Eng. J. 382, 122949. doi:10.1016/j.cej.2019.122949

Yan, M., Liu, Y., Zhu, X., Wang, X., Liu, L., Sun, H., et al. (2019). Nanoscale Reduced Graphene Oxide-Mediated Photothermal Therapy Together with Ido Inhibition and PD-L1 Blockade Synergistically Promote Antitumor Immunity. ACS Appl. Mater. Inter. 11, 1876-1885. doi:10.1021/acsami.8b18751

Yang, Y., Asiri, A. M., Tang, Z., Du, D., and Lin, Y. (2013). Graphene Based Materials for Biomedical Applications. Mater. Today 16, 365-373. doi:10.1016/ j.mattod.2013.09.004

Zhang, Y., Ma, C., Xie, J., Ågren, H., and Zhang, H. (2021). Black Phosphorus/polymers: Status and Challenges. Adv. Mater. 33, 2100113. doi:10.1002/adma.202100113

Zhong, X., Wang, X., Li, J., Hu, J., Cheng, L., and Yang, X. (2021). ROS-based Dynamic Therapy Synergy with Modulating Tumor Cell-Microenvironment Mediated by Inorganic Nanomedicine. Coord. Chem. Rev. 437, 213828. doi:10.1016/j.ccr.2021.213828

Conflict of Interest: The authors declare that the research was conducted in the absence of any commercial or financial relationships that could be construed as a potential conflict of interest.

Publisher's Note: All claims expressed in this article are solely those of the authors and do not necessarily represent those of their affiliated organizations, or those of the publisher, the editors and the reviewers. Any product that may be evaluated in this article, or claim that may be made by its manufacturer, is not guaranteed or endorsed by the publisher.

Copyright () $2021 \mathrm{Zhu}$, Li and Luo. This is an open-access article distributed under the terms of the Creative Commons Attribution License (CC BY). The use, distribution or reproduction in other forums is permitted, provided the original author(s) and the copyright owner(s) are credited and that the original publication in this journal is cited, in accordance with accepted academic practice. No use, distribution or reproduction is permitted which does not comply with these terms. 